\title{
Two-dimensional photonic crystals from semiconductor material with polymer filled holes
}

\author{
Rob van der Heijden ${ }^{* a, b}$, Charlotte Kjellander ${ }^{\mathrm{b}, \mathrm{c}}$, Carl-Fredrik Carlström ${ }^{\mathrm{a}, \mathrm{b}}$, Juri Snijders ${ }^{\mathrm{a}, \mathrm{b}}$, \\ Rob W. van der Heijden ${ }^{\mathrm{a}, \mathrm{b}}$, Kees Bastiaansen ${ }^{\mathrm{c}}$, Dick Broer ${ }^{\mathrm{c}}$, Fouad Karouta ${ }^{\mathrm{a}}$, Richard Nötzel ${ }^{\mathrm{a}, \mathrm{b}}$, \\ Emile van der Drift ${ }^{\mathrm{d}}$, and Huub W.M. Salemink ${ }^{\mathrm{a}, \mathrm{b}, \mathrm{d}}$ \\ ${ }^{a}$ COBRA Inter-University Research Institute and Center for NanoMaterials, \\ Eindhoven University of Technology, PO Box 513, NL-5600 MB Eindhoven, The Netherlands \\ ${ }^{\mathrm{b}}$ Department of Applied Physics, Eindhoven University of Technology, PO Box 513, \\ NL-5600 MB Eindhoven, The Netherlands \\ ${ }^{c}$ Department of Chemistry and Chemical Engineering, Eindhoven University of Technology, \\ PO Box 513, NL-5600 MB Eindhoven, The Netherlands \\ ${ }^{\mathrm{d}}$ Kavli Institute of Nanoscience, Delft University of Technology, P.O. Box 5053, \\ NL-2600 GB Delft, The Netherlands
}

\begin{abstract}
Polymer filling of the air holes of indiumphosphide based two-dimensional photonic crystals is reported. The filling is performed by infiltration with a liquid monomer and solidification of the infill in situ by thermal polymerization. Complete hole filling is obtained with infiltration under ambient pressure. This conclusion is based both on crosssectional scanning electron microscope inspection of the filled samples as well as on optical transmission measurements.
\end{abstract}

Keywords: Infiltration, Photonic crystal, InP

\section{INTRODUCTION}

Photonic crystals (PhCs) are materials consisting of a periodic arrangement of low- and high dielectric constant materials that act in the same way for lightwaves as the periodic potential of a crystal lattice does for electrons in a semiconductor. As a consequence, photonic bandgaps are created, i.e. frequency regions where light cannot propagate in the periodic medium. This property can be exploited to provide control of light at a wavelength scale ${ }^{1}$ and as such photonic crystals will find many applications in photonic circuitry or in quantum-optic devices. Two-dimensional (2D) $\mathrm{PhCs}$ in semiconductor materials are of particular relevance as they can be fabricated starting from the patterning and etching technology that is already available from electronic applications. Confinement of the optical wave to the 2D plane is achieved by a layer structure that acts as a planar waveguide. The InP-material system is relevant for applications at the telecommunication wavelength of $1550 \mathrm{~nm}$.

The application area of $\mathrm{PhC}$ structures will be greatly enhanced when their optical properties are tunable. It was proposed by Busch and John ${ }^{2}$ to replace the low-index part (air) with a liquid crystal (LC) material that has a tunable refractive index. Since then several experiments have been reported both in 3 dimensions (see Ref. 3 for an overview) and in 2D that combine liquid crystals with photonic crystals. ${ }^{4-10}$ The LC tuning is inherently slow, typically in the range of $\mathrm{ms}$ to $\mu \mathrm{s}$. Also, the liquid state could inhibit some applications or limit the compatibility with other processing steps. Therefore it is of interest to investigate other materials, notably electro-optically active or optically non-linear (NLO) polymers for use as a low-index dielectric in the semiconductor host.

*e-mail: r.v.d.heijden@tue.nl

Photonic Crystal Materials and Devices III, edited by Richard M. De La Rue, Pierre Viktorovitch, Ceferino Lopez, Michele Midrio, Proc. of SPIE Vol. 6182, 618214, (2006) · 0277-786X/06/\$15 · doi: 10.1117/12.662660 
Even filling of the holes with a passive dielectric will find several applications. The index contrast between the high and low dielectric is reduced due to the filling. The diffraction angle when the light passes from the waveguiding dielectric into the holes is therefore smaller and out-of-plane scattering is reduced for deeply etched PhCs. A solid infill would allow planarization of the sample surface and further polymer processing may be employed to create additional functionality in the polymer layer on top. If particular holes can be filled selectively, a new tool is available to create components in optical integrated circuits in "bulk" $2 \mathrm{D} \mathrm{PhCs.}{ }^{11}$

In this paper, filling of hole type 2D PhCs in InP with a solid polymer is reported by infiltration of a liquid monomer and subsequent in situ thermal polymerization. In the following, first the relevant experimental conditions are discussed. Then the complete filling is shown directly from cross-sectional scanning electron microscope images. Optical evidence of the filling is provided by the shift of the photonic bandgap observed in transmission spectra of PhCs before and after infiltration.

\section{INFILTRATION PARAMETERS}

Photonic crystals made in the double heterostructure type planar waveguides (InP-InGaAsP-InP) imply etching of $\sim 200$ $\mathrm{nm}$ diameter holes to a depth of more than $2 \mu \mathrm{m}$ in the semiconductor material. The capillary pressure $p_{\text {cap }}$ provides the driving force for the liquid flow and is given by:

$$
p_{c a p}=\frac{2 \gamma \cos (\theta)}{r} .
$$

Here $r$ is the hole radius, $\gamma$ the surface tension of the liquid and $\theta$ the contact angle between the liquid and the solid. For successful infiltration it is crucial that the liquid wets the surface, i.e. $\theta<<90^{\circ}$. The typical surface tension for most liquids is in the order of $10-100 \mathrm{mN} / \mathrm{m}$ (see Ref. 12, table 6-150), implying that the capillary pressure is in the range 2 to $20 \mathrm{bar}$. The surface tension of the monomer used in our experimental work is $36.1 \mathrm{mN} / \mathrm{m}$. If the infiltration is performed under a pressure $p_{0}$, a residual volume $\left(p_{0} / p_{\text {cap }}\right) V_{\text {hole }}$ of compressed air would remain unfilled, provided that the air cannot escape. This is a substantial fraction of the total hole volume $V_{\text {hole }}$ for filling at ambient pressure $p_{0} \sim 1$ bar. In previous work, ${ }^{4-6,9-10}$ infiltration of deeply etched PhCs was performed under vacuum conditions, which means that $p_{0}<<1$ bar.

The air permeability of most liquids would be sufficient for the small holes to degas efficiently under a pressure up to several bars. The degas time can be estimated from the volume gas flow given by ${ }^{13}$ :

$$
\Phi_{\text {gas }}=P \frac{A \Delta p}{h},
$$

where $P$ is the gas permeability of the liquid, $\Delta p$ the pressure drop across the liquid column, and $A$ its cross-sectional area and $h$ the hole depth. The degassing time can then be estimated from $\tau_{\text {degas }} \sim V_{\text {hole }} / \Phi_{\text {gas }}$ with $\Delta p \sim p_{\text {cap. }}$ The exact value of $P$ for the infiltrants used is not known, but the possible range may be obtained using values for the $\mathrm{N}_{2^{-}}$ permeability of polyethylene (PE) ${ }^{13}$ and water (Ref. 12, tables 6-201 and 8-88). This yields hole degassing times in the order of $1 \mathrm{~s}$ for $3 \mu \mathrm{m}$ deep holes for the PE permeability and even two orders of magnitude shorter times when using the water permeability value. It is therefore concluded that the residual air at the bottom of the hole diffuses through the liquid well within the timescale of the infiltration procedure. This result indicates that vacuum conditions are not required for successful infiltration.

A typical time scale for the infiltration can be estimated from the Hagen-Poiseuille flow law for the liquid flowing into the hole, yielding

$$
\tau_{\text {fill }}=\frac{4 \mu h^{2}}{r \gamma \cos (\theta)} .
$$

Here $\mu$ is the viscosity of the liquid, and the pressure gradient is estimated by $p_{\text {cap }} / h$. The liquid monomer used in our experimental work has a viscosity $\sim 150 \mathrm{mPa} \cdot \mathrm{s}$ so that the infiltration time for holes of $\sim 3 \mu \mathrm{m}$ deep would be $\sim 1 \mathrm{~ms}$. Even very viscous liquids (e.g. glycerine with $\mu \sim 1 \mathrm{~Pa} \cdot \mathrm{s}$ ) yield fill times $<<1 \mathrm{~s}$, short compared to the time scale of the infiltration. From this it follows that viscosity should not be critical for the infiltration. 


\section{INFILTRATION EXPERIMENTS}

\subsection{Procedure}
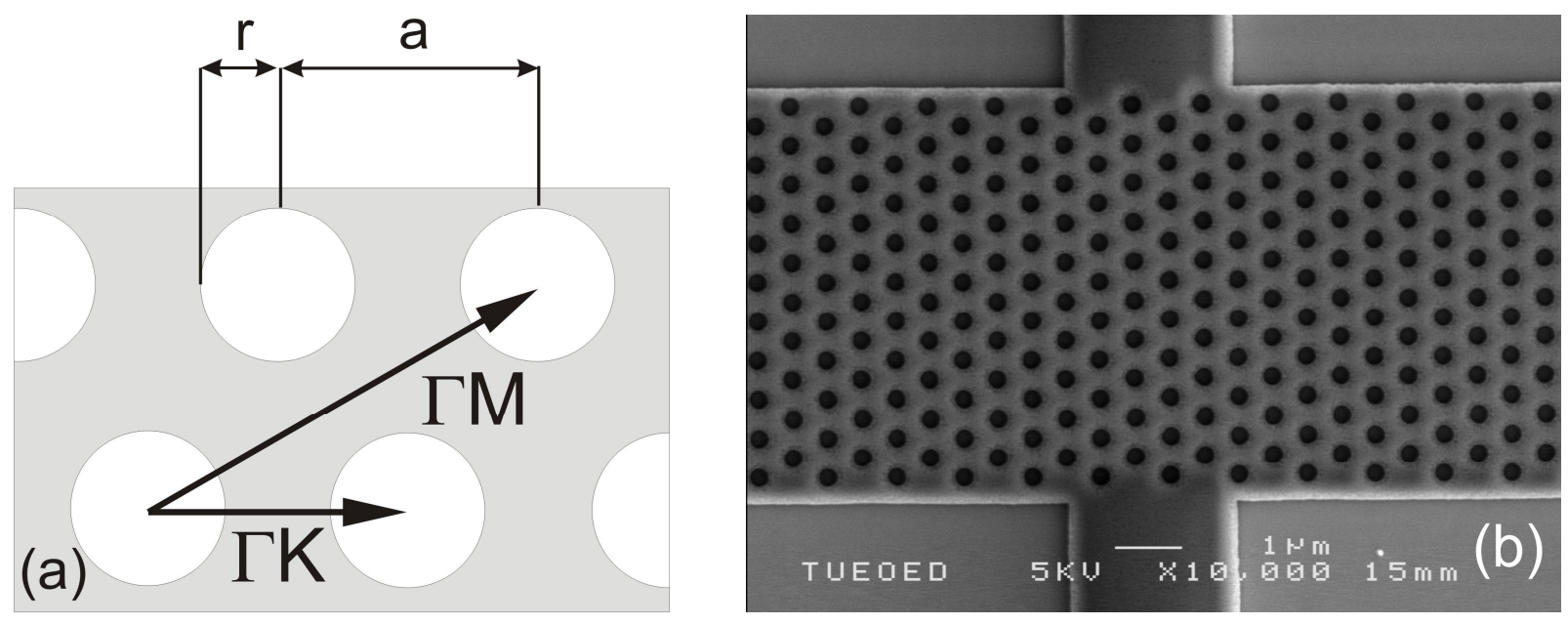

Figure 1: (a) Schematic top view of a triangular lattice of air holes. The two symmetry directions ( $Г K$ and $\Gamma \mathrm{M})$ and lattice parameters (hole radius $r$ and lattice constant $a$ ) are depicted in the drawing. (b) Scanning electron microscope top view of a fabricated $\mathrm{PhC}$. Ridge waveguides are visible at the top and bottom of the $\mathrm{PhC}$ field.

Triangular lattice photonic crystal patterns were fabricated in InP substrates using $100 \mathrm{keV}$ electron-beam lithography, reactive ion etching of a $\mathrm{SiN}_{\mathrm{x}}$ hard mask and $\mathrm{Cl}_{2} / \mathrm{O}_{2}$-based inductively coupled plasma etching ${ }^{14}$ (see Figure 1). The patterns were filled with polymer by infiltration with a liquid monomer and subsequent thermal polymerization. Trimethylolpropane triacrylate (TMP-3A, Aldrich) was chosen as the infiltrant for its good cross-linking efficiency. A fraction of $0.5 \mathrm{wt}$ \% azo-bisisobutyronitrile (AIBN, Fluka) is added to the TMP-3A as initiator for the thermal polymerization process. Before infiltration, the wetting of the InP surface by the liquid monomer was directly investigated by contact angle measurements. The surface was prepared by rinsing with several anorganic acids or organic liquids and the resulting contact angle was subsequently measured. The best result was obtained after a successive rinse with $10 \%$ hydrofluoric acid in water and propanol-2, which reduced the contact angle from $33^{\circ}$ to $<4^{\circ}$. A droplet of the monomer was spread onto the optimally prepared surface, completely covering the hole pattern. Experiments were done with depositing the droplet under vacuum $(20 \mathrm{mbar})$ conditions under under ambient atmosphere. The thermal polymerization is performed on a hotplate. The heating was performed under nitrogen gas flow conditions, to minimize oxygen reaction with the radicals formed. To suppress formation of cracks in the polymer, the temperature is gradually increased from room temperature up to $50{ }^{\circ} \mathrm{C}$. The liquid is then allowed to polymerize for 10 minutes, with a subsequent increase in temperature to $70{ }^{\circ} \mathrm{C}$ in order to enhance the mobility of the unreacted monomers. The sample was maintained at this temperature for 20 minutes to complete the polymerization.

The refractive index of the polymer was measured in a separate experiment using a spectroscopic ellipsometer. For this purpose it was necessary to spin-cast a thin layer on a Si-substrate and subsequently it was given the same heating treatment as the infiltrated samples. For wavelengths around $1550 \mathrm{~nm}$ the measured refractive index is $1.465 \pm 0.005$; no absorption in this wavelength region was measurable with this spectrometer.

\subsection{Scanning electron microscope results}

The filled hole pattern is inspected with cross-sectional scanning electron microscopy (SEM) using a cleaved sample. To ensure that the cleave intersects the holes, the $Г \mathrm{~K}$ or $Г \mathrm{M}$-axis of the photonic crystal were oriented under a small angle $\left(\sim 3^{\circ}\right)$ with respect to the InP crystal cleavage directions. In Figure 2 a SEM view of the holes is shown after infiltration in (a) vacuum (20 mbar) and (b) ambient atmosphere. The holes are filled down to the bottom irrespective of 
the filling conditions, which supports the conclusion of section 2. At some locations, it appears that the polymer plug becomes detached from the sidewalls, as may be inferred from the right side of the second hole



Figure 2: SEM view of the photonic crystal holes after infiltration (a) in vacuum and (b) ambient atmosphere with TMP-3A. These holes are etched in InP substrate with a non-optimized ICP-process that results in a tapered profile. The polymer layer on top is visible for both samples.

from the left in Fig. 2(b). The detachment is attributed to the polymerization shrinkage, which is expected to be $10-15 \%$ for poly-acrylates. Some holes in Figure 2 appear to be empty. This is attributed to the non-cleaving of the polymer plugs that thus remain on either side of the cleavage.

In one experiment, both halves of the cleaved samples were inspected in the SEM. The same holes were identified on both sides. The polymer plug was found back for every hole, either in one or in the other halve of the cleaved sample. These experiments were carried out on samples used for testing different etch processes and therefore the hole shapes vary. In all cases investigated, the polymer was observed to fill the holes down to the bottom, even for the irregular and conical hole shapes as in Figure 2.

\section{OPTICAL MEASUREMENTS}

\subsection{Sample structure}
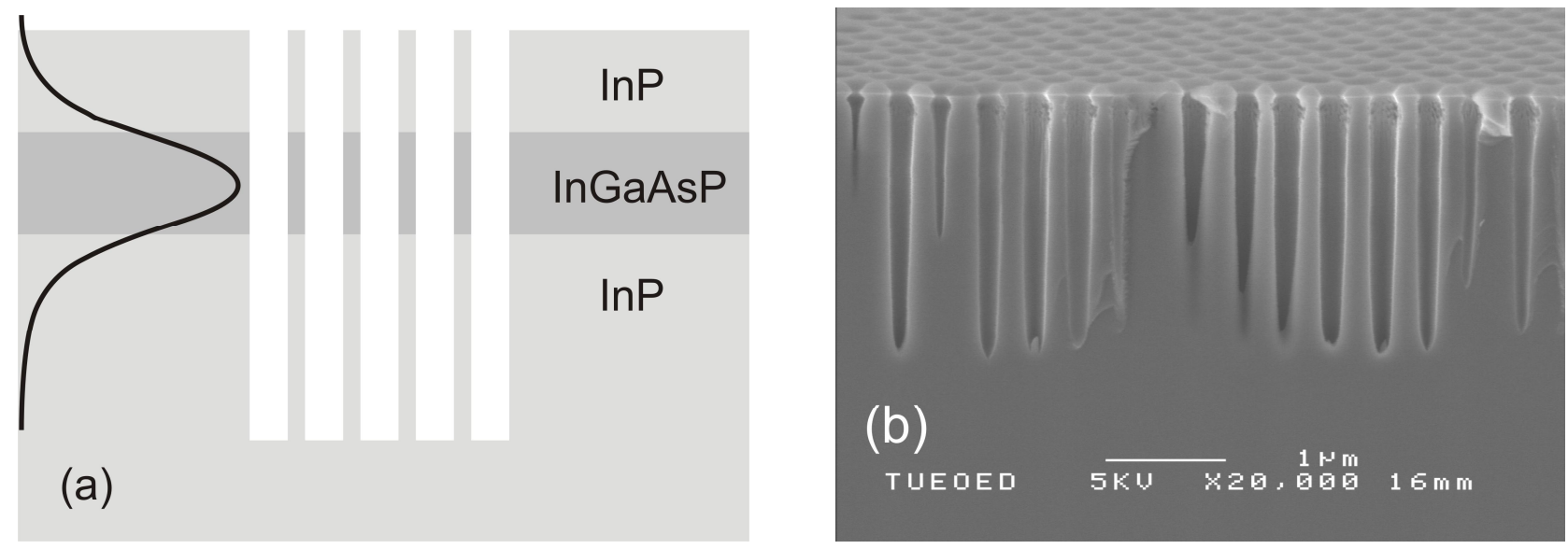

Figure 3: (a) Schematic side view of the planar waveguide sample used for optical measurements. The intensity profile of the TEguided mode for a wavelength of $1550 \mathrm{~nm}$ is shown on the left. (b) SEM view of the hole profile of an InP sample etched in the same run as the sample that was optically characterized.

To optically characterize the effect of the filling on the photonic bandgap, samples were prepared with a 10-period triangular lattice of air holes in the $\Gamma \mathrm{K}$-direction with an air-filling factor of 0.33 . 
For these optical test samples, the holes were etched through an $\mathrm{InP} / \mathrm{InGaAsP} / \mathrm{InP}$ planar waveguide structure. Both the InGaAsP (lattice matched, band-edge wavelength $\lambda=1.25 \mu \mathrm{m}$ ) and the InP upper cladding are $500 \mathrm{~nm}$ thick, which leads to an effective refractive index of 3.25 for the transverse electric (TE) guided mode at a wavelength of $1550 \mathrm{~nm}$. The intensity profile of this guided mode is shown schematically in a side view of the waveguide structure in Figure $3 \mathrm{a}$. The ridge waveguides that access the photonic crystal (see Figure 1b) are produced in the same etching step as the photonic crystal holes. The optimum ICP-etching processes for making high-aspect ratio holes or smooth waveguides are very different. Therefore for the test samples a process compromising between the hole shape and ridge waveguide shape had to be used.

An InP reference sample, suitable for SEM inspection, was etched simultaneously with the planar waveguide sample. A cross-sectional SEM-view of the vertical hole-profile for this reference sample is shown in Figure $3 \mathrm{~b}$. In a separate series of etch tests it was verified that hole shapes etched in heterostructure waveguide material and in pure InP are similar for the etch process used. Ridge waveguides with optical quality end facets were obtained by cleaving the sample perpendicular to the ridges.

\subsection{Measurement set-up}

The waveguides are excited with light from a tunable $(1470-1570 \mathrm{~nm})$ polarization controlled diode laser. TE-polarized (E-field in the photonic crystal plane) radiation is used only. Coupling in and out of the waveguides is accomplished with microscope objectives (N.A. $=0.65$ ). The beam is chopped and the transmitted light is measured with an InGaAs photodetector using a lock-in amplifier. Compiling transmission spectra from a series of photonic crystals with slightly varying lattice constants provided the full coverage of the $\Gamma \mathrm{K}$ stopband of the photonic crystal in dimensionless frequency units $a / \lambda$. First, the transmission data were collected from the samples as etched. After that, the holes were infiltrated with TMP-3A under atmospheric conditions. A thick polymer droplet remained on the surface after polymerization. Under SEM inspection, no contamination of the end facets with the polymer could be observed. The transmission spectra for the empty and filled PhCs are given in Figure 4. Each spectrum consists of a compilation of data from $17 \mathrm{PhCs}$.

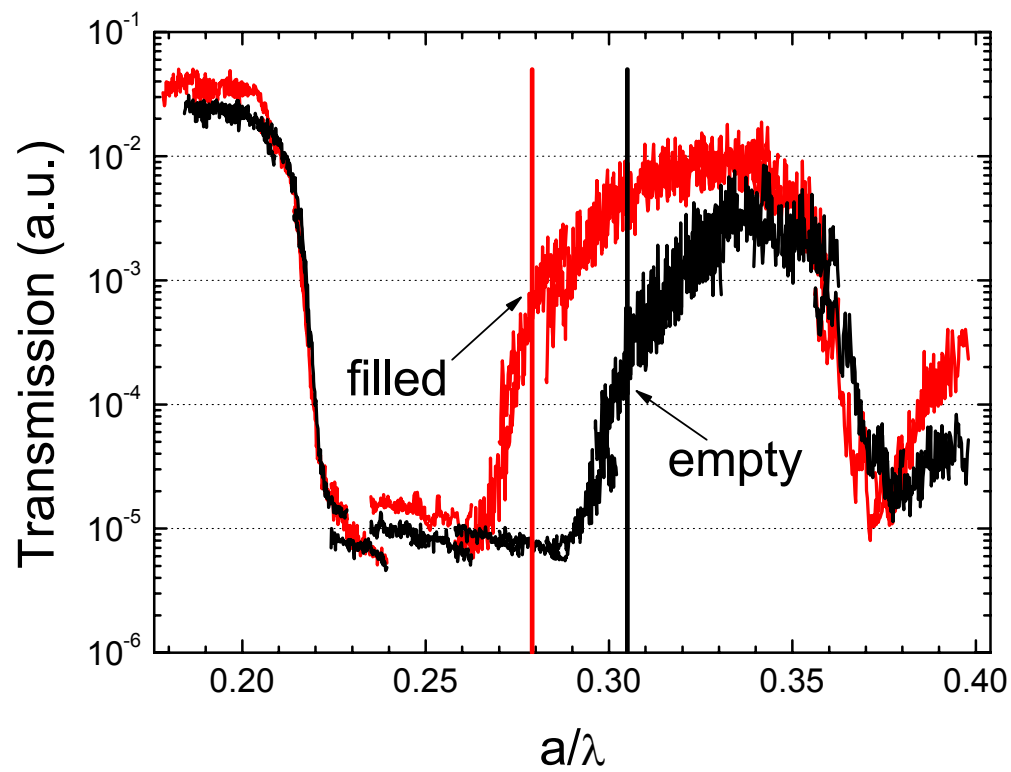

Figure 4: Measured transmission spectrum of an empty and infiltrated triangular lattice 2D photonic crystal with 10 periods in the $\Gamma K$-direction obtained by lithographic tuning (air filling factor $=0.33$ ). The vertical lines represent the calculated position of the band edges for both empty and fully infiltrated holes. 
For the empty structure, the measured stopband extends from $a / \lambda=0.22$ to $a / \lambda=0.30$, where $a$ is the lattice constant of the $\mathrm{PhC}$ and $\lambda$ the wavelength. These $a / \lambda$ values are in agreement with band structure calculations employing a 2D plane wave method ${ }^{15}$ as shown by the vertical lines in Figure 4 . At the low-frequency band edge the transmission rapidly drops by more than three orders of magnitude. The transmission level inside the stopband is determined by stray light reaching the detector and is not the intrinsic level. The high-frequency band edge is not as steep as the low-frequency one, which is attributed to out-of-plane losses ${ }^{16}$. At the calculated position of the high-frequency band edge, the transmission level is $10 \%$ of the maximum transmission in that band. The minimum around $a / \lambda \sim 0.37$ corresponds to higher frequency band structure effects and is known as a pseudo gap. The reduced transmission level in the highfrequency band compared to the lower one is attributed to the finite etch depth and non-cylindrical profile of the holes. ${ }^{16}$

After infiltration, the high-frequency band edge is shifted to the lower frequency $a / \lambda=0.285 \pm 0.05$, whereas the shift for the low-frequency band edge is negligible. This results from the preferred localization of the light in the low refractive index material for the high-frequency band. From the 2D plane wave calculation an accurate dependence of the band edge on the refractive index inside the hole was established. This relation was used to obtain an effective index $n_{\text {hole }}$ from the measured shift of the edge before and after filling. Assuming that the effective index measures the filling fraction, the filling efficiency $\eta$ is calculated via: $\eta=\left(n_{\text {hole }}-1\right) /\left(n_{\text {polymer }}-1\right)$, where $n_{\text {polymer }}$ is the refractive index of the polymer $^{6}$. The accuracy of this method is not very high but it leads to a lower bound on the filling efficiency of $\eta \geq 0.8$. This is consistent with the complete infiltration of the holes by the liquid and allows for a polymerization shrinkage of 10-15\%. From Figure 4 it can also be concluded that the transmission after infiltration is higher in both the bands, the increase being larger for the high-frequency band. It is verified, that the transmission changes of ridge waveguide, which is also immersed in the polymer, are an order of magnitude less than the observed changes in Figure 4. These results indicate that reducing the in-plane index contrast of reduces the out-of-plane losses. This effect is largest for the high-frequency band as this is most sensitive to out-of-plane losses ${ }^{16}$.

\section{CONCLUSION}

We have fabricated 2D photonic crystals in InP and filled the air holes with a solid polymer by infiltration with a monomer and in situ polymerization. The infiltration relies on the capillary pressure of the liquid monomer inside the holes. It is therefore crucial that the liquid wets the surface. Infiltration and the diffusion of the residual air under the fluid column occur well within the timescale of our experiment, which implies that vacuum conditions are not required for full infiltration. Cross-sectional SEM inspection of the samples after polymerization of the infill confirms that the holes are filled down to the bottom for both vacuum and ambient conditions. Optical transmission measurements of samples infiltrated at ambient conditions show that the filling efficiency is $>80 \%$. This number is consistent with complete infiltration of the holes followed by polymerization shrinkage of $10-15 \%$. The filling procedure presented should also be useful with optically active polymers to provide electro-optical tunability of the PhC structures. When applied to selective filling of particular holes, it opens the way for $\mathrm{PhC}$ components with adjustable or switchable optical properties.

\section{ACKNOWLEDGEMENTS}

The authors would like to thank P.Nouwens, R. van Veldhoven, E.J. Geluk, T. de Vries, M. Sander, and E. Smalbrugge for their contributions to the experimental work and J. van der Tol, A. Kok, S. Oei, J. Haverkort, and A. Silov for helpful discussions. Part of this research is supported by NanoNed, a technology programme of the Dutch ministry of Economic Affairs.

\section{REFERENCES}

\footnotetext{
${ }^{1}$ J.D. Joannopoulos, R.D. Meade, and J.N. Winn, Photonic crystals, (Princeton University Press, 1995).

${ }^{2}$ K. Busch, and S. John, Phys. Rev. Lett. 83, 967 (1999).

${ }^{3}$ S. Gottardo, D.S. Wiersma, and W.V. Vos, Physica B 338, 143 (2003).

${ }^{4}$ S.W. Leonard, J.P. Mondia, H.M. van Driel, O. Toader, S. John, K. Busch, A. Birner, U. Gösele, and V. Lehman, Phys. Rev. B 61, R2389 (2000).

${ }^{5}$ Ch. Schuller, F. Klopf, J.P. Reithmaier, M. Kamp, and A. Forchel, Appl. Phys. Lett. 82, 2767 (2003).
} 
${ }^{6}$ J. Martz, B. Wild, R. Ferrini, L.A. Dunbar, M. Mulot, S. Anand, R. Houdré, and L. Zuppiroli, Proc. of SPIE 5926, 592601, (2005).

${ }^{7}$ B. Maune, M. Lončar, J. Witzens, M. Hochberg, T. Baehr-Jones, D. Psaltis, A. Scherer, and Y. Qiu, Appl. Phys. Lett. 85, 360 (2004).

${ }^{8}$ B. Maune, J. Witzens, T. Baehr-Jones, M. Kolodrubetz, H. Atwater, A. Scherer, R. Hagen, and Y. Qiu, Optics Express 13, 4699 (2005).

${ }_{9}^{9}$ G. Mertens, T. Röder, H. Matthias, H. Marsmann, H.S.R. Kitzerow, S. Schweizer, C. Jamois, R.B. Wehrspohn, and M. Neubert, Appl. Phys. Lett. 83, 3036 (2003).

${ }^{10}$ Ch. Schuller, J.P. Reithmaier, J. Zimmermann, M. Kamp, A. Forchel, and S. Anand, Appl. Phys. Lett. 87, 121105 (2005).

${ }^{11}$ S. Mingaleev, M. Schillinger, D. Hermann, and K. Busch, Opt. Lett. 29, 2858 (2004).

${ }^{12}$ Handbook of Chemistry and Physics $84^{\text {th }}$ edition, (CRC press, 2003)

${ }^{13}$ A.F. Stancell, chapter 12 of Polymer Science and Materials, ed. A.V. Tobolsky, and H.F. Mark (Wiley-Interscience, 1971).

${ }^{14}$ C.F. Carlström, R. van der Heijden, F. Karouta, E. van der Drift, R.W. van der Heijden, H.W.M. Salemink, J. Vac. Sci. Technol. B 24, L6 (2006).

${ }^{15}$ M. Plihal and A.A. Maradudin, Phys. Rev. B 44, 8565 (1991).

${ }^{16}$ R. Ferrini, R. Houdré, H. Benisty, M. Qiu, J. Moosburger, J. Opt. Soc. Am. B 20, 469 (2003). 